\title{
Surviving a Single Ventricle and a Single Atrium with a Situs Inversus Viscerum
}

\author{
Randa Tabbah, $M D^{1^{*}}$, Rachoin Rachoin, $M D^{2}$, Bassam Harb, $M D^{3}$ and Kabalan Saroufim, $M D^{4}$
}

${ }^{1}$ Department of Cardiology, Holy Spirit University, Kaslik, Lebanon

${ }^{2}$ Head of the Department of Echocardiography, Notre Dame de Secours University Hospital, Lebanon

${ }^{3}$ Interventional Cardiologist, Notre Dame de Secours University Hospital, Lebanon

${ }^{4}$ Pulmonary and Critical Care Medicine CHU-NDS Jbeil University Hospital, USEK, Lebanon

*Corresponding author: Randa Tabbah, MD, Department of Cardiology, Holy Spirit University, Kaslik, Lebanon

\begin{abstract}
A single ventricle is a congenital malformation including heart defects that results in having one functional ventricle and another small or hypoplastic one. A new concept of this congenital anomaly defines it as two ventricles unable to sustain separately systemic and pulmonary circulation.

We report a rare case of an 18-years-old female surviving a single ventricle malformation associated with a single atrium and a situs inversus viscerum. She was misdiagnosed in utero and at birth and lost to follow up since her early age when diagnosed at 4-years-old and didn't undergo any palliative surgeries after then due to financial issues. Patient was admitted for heart failure decompensation with hepatic congestion, supraventricular arrhythmias and bilateral pleural effusion and was treated symptomatically. This described patient is a very rare congenital association with a bad prognosis and low survival rate.

Early diagnosis and follow up in these congenital cases are important to improve quality of life and life expectancy.
\end{abstract}

\section{Introduction}

A single ventricle is a congenital malformation including heart defects that results in having one functional ventricle and another small or hypoplastic ventricle [1-3]. A new concept of this congenital anomaly defines it as two ventricles unable to sustain separately systemic and pulmonary circulation. These hearts are not amenable to biventricular repair $[4,5]$. There is no specific or known cause for this anomaly, however fetal ultrasound is a common diagnostic tool in this case. It is defined by a mixture of both oxygenated and poorly oxygenated blood pumped from a unique ventricle so newborns may appear cyanotic with pale extremities $[2,3]$.

Under this anomaly we have different types of malformations [6]. Newborns with restricted pulmonary flow as in pulmonary stenosis survive using a patent ductus arteriosus (PDA) and start to have respiratory distress after its closure and will need surgical procedures to create a PDA like shunt. On the other hand, newborns with coarctation and narrowing of the aorta will have pale extremities and not enough blood pumped to the body surgical procedure as the Norwood is mandatory. In addition, a group of newborns may have no limitation of blood flow to the lungs. After birth, a decrease in pulmonary pressures is seen physiologically, so it's easier to the blood to flow to the lungs. The heart has to maintain a good amount of blood to the body and need to pump 3 to 5 times more blood to compensate for the pulmonary flow, so a band to restrict flow from the pulmonary artery is essential [7]. The well balanced newborns don't require surgery at early age. Glenn surgery then Fontan surgery will be done later [8-10].

The Norwood procedure consist of an atrial septectomy with transaction and ligation of distal main pulmonary artery. The proximal pulmonary artery is connected to the hypoplastic aortic arch and reparation of the coarcted aortic segment is required. Pulmonary blood flow is maintained via a modified Blalock-Taussig shunt.

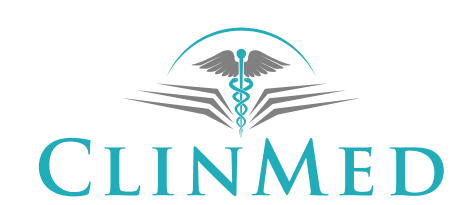

INTERNATIONAL LIBRARY

Citation: Tabbah R, Rachoin R, Harb B (2019) Surviving a Single Ventricle and a Single Atrium with a Situs Inversus Viscerum. Clin Med Rev Case Rep 6:278. doi.org/10.23937/2378-3656/1410278 Accepted: August 22, 2019: Published: August 24, 2019

Copyright: (C) 2019 Tabbah R, et al. This is an open-access article distributed under the terms of the Creative Commons Attribution License, which permits unrestricted use, distribution, and reproduction in any medium, provided the original author and source are credited. 
The Glenn procedure involve a separation of the systemic and pulmonary circulation followed by a removing of the aorto-pulmonary shunt pursued by a creation of a bidirectional superior vena cava and pulmonary shunt. Finally the Fontan procedure at 2 to 4 years of age consist of connecting the inferior vena cava to the branch of the pulmonary artery $[5,9,10]$. Palliative surgeries are mandatory for a better survival.

\section{Clinical Case}

We report a rare case of an 18-years-old female misdiagnosed in utero and at birth for having a congenital cardiac defect. Birth was normal with no complications nor prematurity. She was lost to follow up since her early age when diagnosed at 4-years-old with a rare congenital anomaly and didn't undergo any palliative surgeries after then due to financial issues. Child remain asymptomatic complaining sometimes for dyspnea on severe physical effort.
The young female complained of non-specific symptoms and was admitted in another hospital for tachycardia and abdominal pain. An electrocardiogram (EKG) was done revealing a narrow complex tachycardia (AVNRT: Atrioventricular nodal reentry tachycardia) back to sinus after $300 \mathrm{mg}$ of amiodarone. Patient was transferred to our facility for further investigation.

At presentation, she had dyspnea at rest with palpitation and a right upper quadrant pain. On inspection she was cyanotic with clubbing. Physical exam revealed, clear lungs with a tenderness on the right upper quadrant pain and mild jugular venous distension with bilateral lower limb edema. On auscultation, a systolic crescendo-decrescendo murmur was discovered with maximal intensity at mid systole best heard at the left lateral sternal border. Chest radiography revealed cardiomegaly with an increase in pulmonary vascular bed (Figure 1).

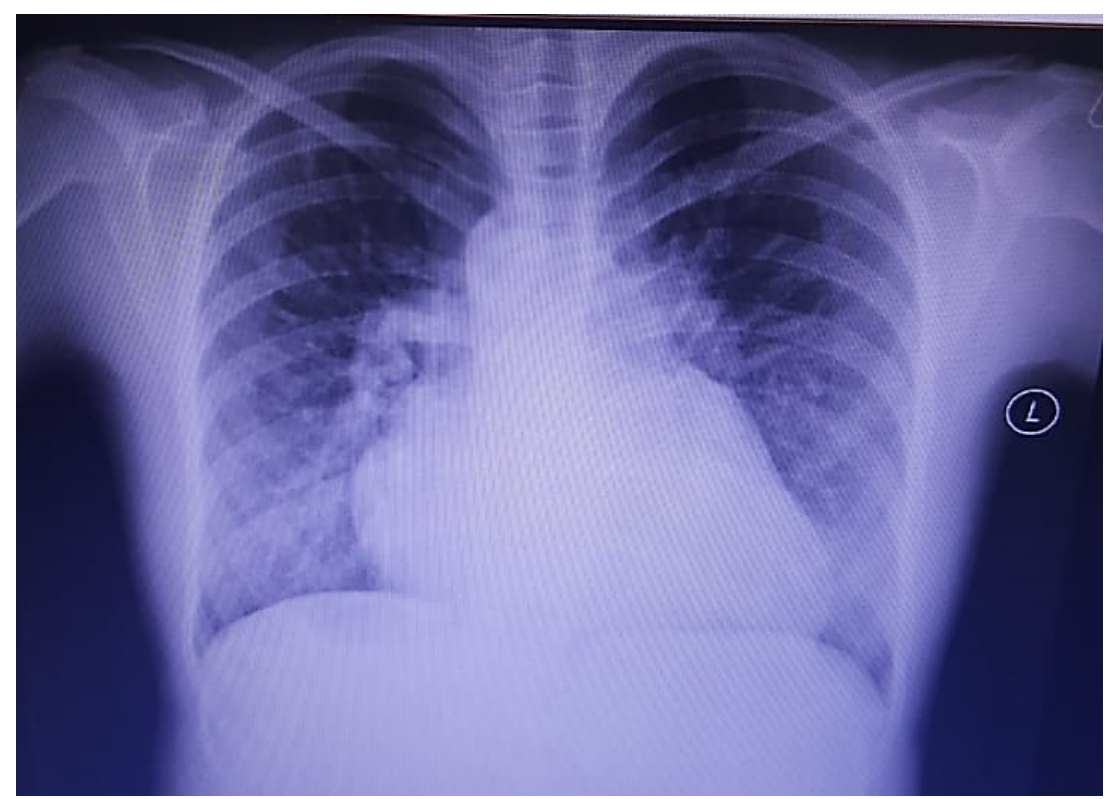

Figure 1: Chest radiography: Enlarged cardiac silhouette with an increase in pulmonary vascular bed.

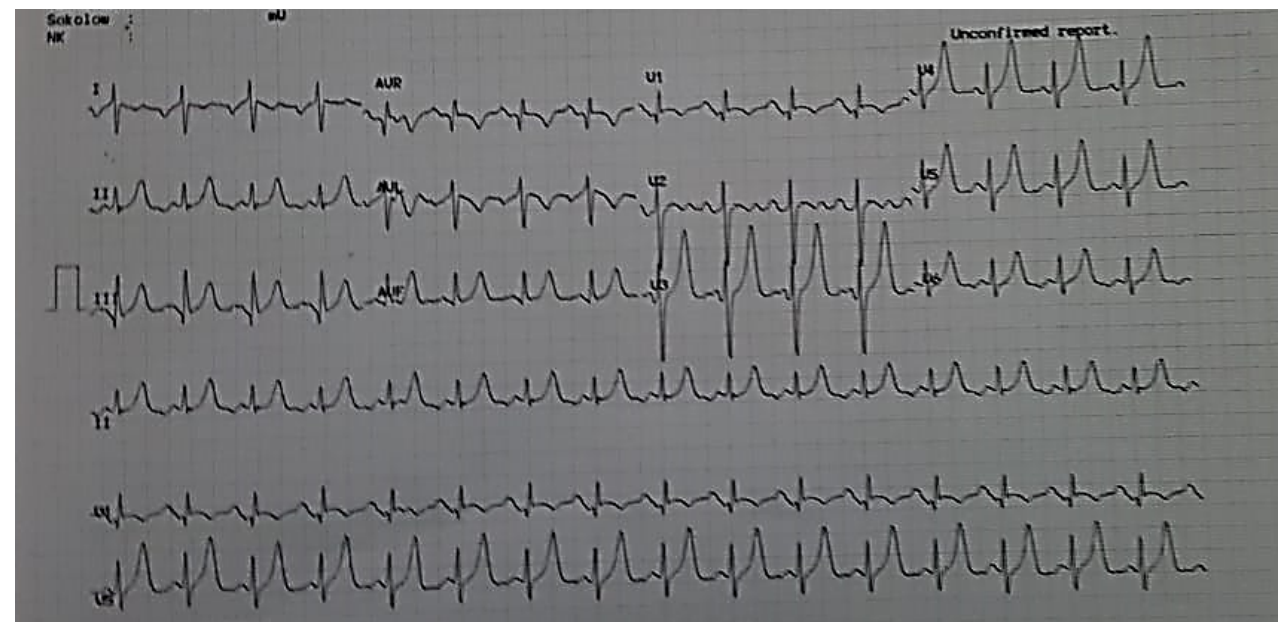

Figure 2: Electrocardiogram: Sinus rhythm with right axis deviation and an incomplete right bundle branch block with a peaked T wave from $\mathrm{v} 3$ to $\mathrm{v} 6$. 
EKG revealed a sinus rhythm with right axis deviation and an incomplete right bundle branch block with a peaked T wave from v3 to v6 (Figure 2). Lab tests reported normal values of cardiac enzymes with normal creatinine levels and high hepatic enzymes levels Serum glutamic oxaloacetic transaminase $(\mathrm{SGOT})=431 \mathrm{U} / \mathrm{L}$ and Serum glutamic pyruvic transaminase (SGPT) $=357$ $\mathrm{U} / \mathrm{L}$ ), with a high international normalized ratio (INR) = 2.31, Brain natriuretic peptide (BNP) levels $=3681 \mathrm{pg} /$ $\mathrm{ml}$ and Hemoglobin $=16.7 \mathrm{~g} / \mathrm{dl}$ and Hematocrit $=55.7$.

The assessment of this congenital anomaly was done with a sequential segmental approach. Cardiac ultrasound revealed a unique large atrium "core biloculare" [4] with a concordant usual arrangement and a unique large ventricle with fine apical trabeculations of left ventricular morphology with an anterior position of the hypoplastic right ventricle [5] and a reduced systolic function on two-dimensional 2D ejection fraction of $35 \%$. A common atrioventricular valve with severe eccentric regurgitation grade 3 on four chamber view is seen (single inlet). A tricuspid atresia is identified. Furthermore, the two major arteries arise from the single ventricle. It's a double outlet ventricle. An aorta arising from the morphological left ventricle with an aortic regurgitation grade 1 to 2 mostly seen on parasternal long axis (Figure 3). No coarctation of the aorta on suprasternal view. A narrowed main pulmonary artery is recognized emerging from the unique ventricle with an infundibular stenosis and a peak gradient of 85 $\mathrm{mmHg}$. Two normal right and left pulmonary branches arise from the main pulmonary artery. Furthermore, ultrasound revealed a congested left liver with a dilated vena cava and hepatic veins. A computerized tomography revealed a bilateral pleural effusion and ascites. A pure organ situs inversus viscerum is noticed with a congested left liver and a right stomach. To the right, polysplenia is seen as an incidental finding. No dextrocardia is recognized nor pulmonary and bronchial malformations.

Patient was treated conservatively with furosemide and eplerenone for symptoms relieve, beta-blockers (bisoprolol) for supraventricular arrhythmias and rate control, amiodarone due to some episodes of paroxysmal atrial fibrillation and an angiotensin inverting enzymes inhibitors was added to the treatment for his effect on morbidity and mortality in heart failure patients and it may be associated with a reduction in renal injury, improvement in neurodevelopment outcomes and endothelial function in single ventricle patients. She didn't tolerated this medication due to her low blood pressure [11-14].

This patient presented with natural course of the complications of single ventricle with long term survival with no surgeries and medical therapies. Severe heart failure and supraventricular arrhythmias were seen. Patient refused any palliative surgery at this state due to their high risk and comorbidities.

The physician sent her for transplantation purposes. Patient on transplantation list waiting for a new heart.

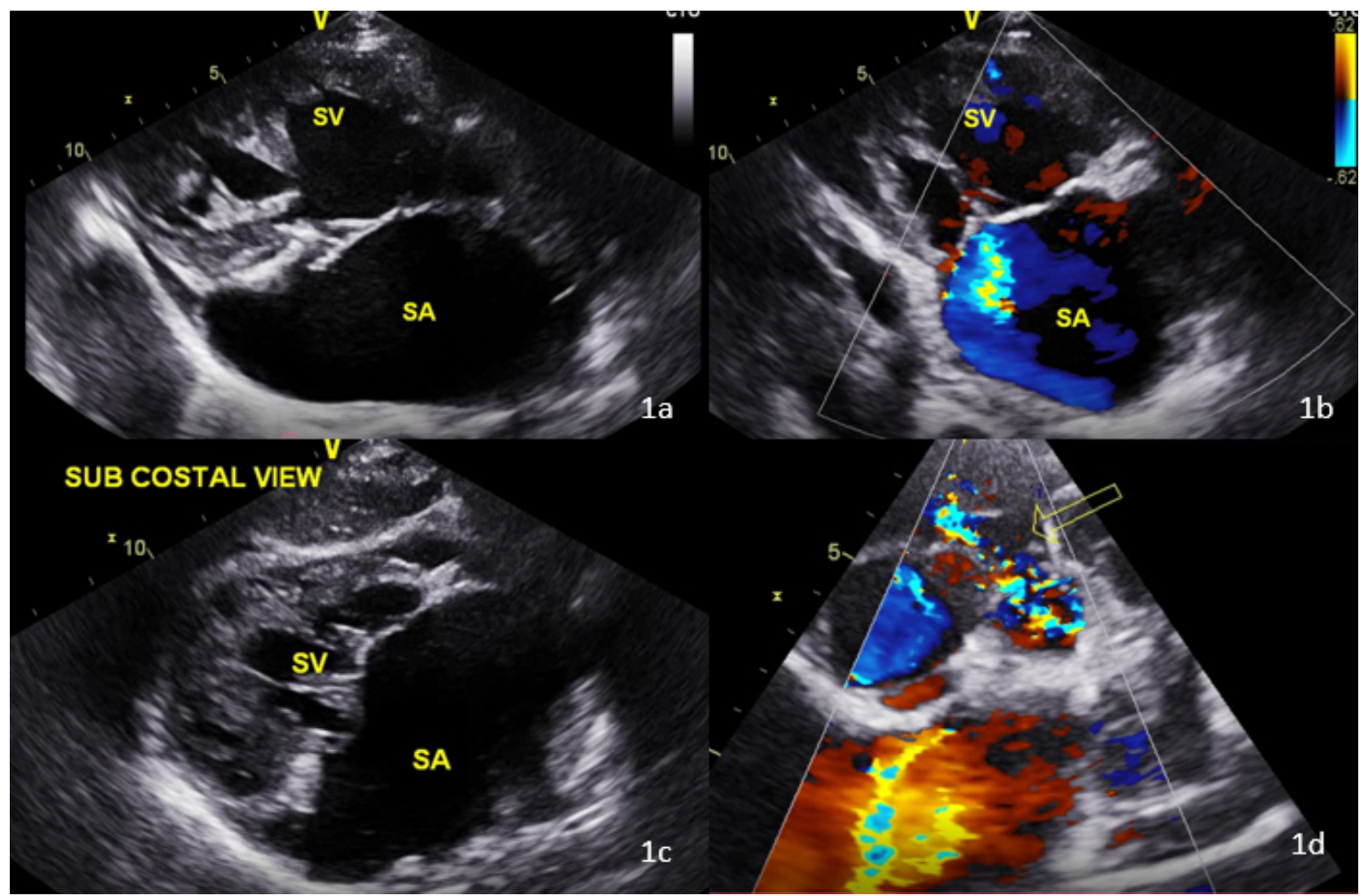

Figure 3: Echocardiographic finding in single ventricle patient.

a) Four chamber view: Single ventricle and single atrium; b) Four chamber view: Eccentric regurgitation of the atrio-ventricular valve; c) Subcostal view: Single ventricle and single atrium; d) Parasternal short axis view: pulmonary Infundibular stenosis. 


\section{Discussion}

This described patient didn't receive any palliative surgery since her diagnosis and she was lost to follow up surviving till 18-years-old with some dyspnea on exertion and chronic cyanosis with clubbing. In adults there is a higher surgical risk (10\%) due to long lasting overloads in addition to ventricular dysfunction and anatomical lesions to the valves so the pros and cons for palliative surgical treatment are needed to be discussed in this case. This risk was discussed with the patient who refused further surgical management. In the normal course for surgical treatment of these patients the entity with a high pulmonary flow will eventually need banding procedures, on the other hand, those with a reduced pulmonary blood flow will need a Blalock-Taussig shunt followed by Glen procedure then at 2 to 3 years old fontan procedure. In this case, a pulmonary infundibular stenosis was compensatory and compatible with life.

In our case, the patient has also a single atrium which is a very rare variety characterized by an embryological deficient interatrial septum "core biloculare". The association single atrium and single ventricle is very uncommon which result a mixing of venous and arterial blood causing severe cyanosis in these patients $[1,15]$. The prognosis of patients with single atrium is very poor with a rate of mortality up to $50 \%$. The median age of survival in a patient with a right ventricle morphology is $4 \%$, however the LV morphology is 14 years $[1,16,17]$. Our patient survived till 18-years-old with no palliative surgery nor medical evaluation. Furthermore, we have a very rare association maybe a unique one between a rare condition as a single ventricle and another rare condition the situs inversus viscerum.

The situs inversus viscerum is very rare. This entity was described in cases associated with dextrocardia, but association with levocardia is extremely rare.

Almost $100 \%$ of cases of situs inversus with levocardia are associated with congenital heart disease, most of which are severe or associated with asplenia or polysplenia syndromes. The incidence of these associated anomalies is $1: 22,000$ in the general population. The prognosis is poor, and only $5 \%-13 \%$ of patients survive for more than five years [18]. The patient described in this article has a situs inversus viscerum with normal pulmonary and bronchial morphology with an incidental finding of polysplenia was associated also.

Moreover, patients with congenital anomalies as in this case have a lot of complications in their normal course of life. Atrial tachyarrhythmias are prevalent in this context and challenging. This anomaly is often associated with thromboembolic and hepatic dysfunction as in our patient. Hepatic congestion was noticed and it's not rare in this context [19-21]. Worsening cyanosis is associated with ventricular failure with or without $\mathrm{AV}$ regurgitation with a worsening of exercise tolerance and quality of life and repeated hospital admissions and comorbidities $[4,22]$. The patient we described has an associated very rare anomalies a single ventricle with a single atrium and a situs inversus viscerum but managed to survive till 18-years-old with no surgery nor medication because patient was lost to follow up with a poor family condition. An association of these anomalies is related with poor outcomes and high risk of mortality.

\section{Conclusion}

We presented a case of an association of multiple serious anomalies in a patient surviving till 18 -years-old with no surgery or medical follow up. This patient had a single ventricle and a single atrium with a situs inversus viscerum. The most important things in these anomalies is early diagnosis and follow up of these patients to access the required surgeries and close monitoring of these patients.

\section{References}

1. Patra S, Agrawal N, Usha MK, Jayaranganath M (2013) Common atrium with single ventricle: $A$ rare combination of two uncommon complex congenital heart diseases. BMJ Case Rep 2013.

2. Jacobs ML, Anderson RH (2006) Nomenclature of the functionally univentricular heart. Cardiol Young 16: 3-8.

3. (2015) Atlas of Congenital Heart Disease Nomenclature: An Illustrated Guide to the Van Praagh and Anderson Approaches to Describing Congenital Cardiac Pathology Paperback.

4. Khairy P, Poirier N, Mercier LA (2007) Univentricular heart. Circulation 115: 800-812.

5. Frescura C, Thiene G (2014) The new concept of univentricular heart. Front Pediatr 2: 62.

6. Rahimtoola SH, Ongley PA, Swan HJ (1966) The hemodynamics of common (or single) ventricle. Circulation 34: 14-23.

7. Bittle GJ, Wehman B, Karathanasis SK, Kaushal S (2017) Clinical progress in cell therapy for single ventricle congenital heart disease. Circ Res 120: 1060-1062.

8. Anderson RH, Franklin RCG, Spicer DE (2018) Anatomy of the functionally univentricular heart. World $\mathrm{J}$ Pediatr Congenit Heart Surg 9: 677-684.

9. Jacobs ML, Mayer JE Jr (2000) Congenital heart surgery nomenclature and database project: Single ventricle. Ann Thorac Surg 69: S197-S204.

10. Tynan MJ, Becker AE, Macartney FJ, Quero-Jimenez M, Shinebourne EA, et al. (1979) Nomenclature and classification of congenital heart disease. Br Heart $\mathrm{J} 41$ : 544-553.

11. Kouatli AA, Garcia JA, Zellers TM, Weinstein EM, Mahony $L$ (1997) Enalapril does not enhance exercise capacity in patients after Fontan procedure. Circulation 96: 1507-1512.

12. Hsu DT, Zak V, Mahony L, Sleeper LA, Atz AM, et al. (2009) Enalapril does not improve growth or ventricular function in infants with single ventricle: A multicenter clinical trial. Circulation 120: S560-S561.

13. Jin SM, Noh Cl, Bae EJ, Choi JY, Yun YS (2007) Impaired vascular function in patients with Fontan circulation. Int $\mathrm{J}$ Cardiol 120: 221-226. 
14. Anne P, Du W, Mattoo TK, Zilberman MV (2009) Nephropathy in patients after Fontan palliation. Int $\mathrm{J}$ Cardiol 132: 244-247.

15. Nabati M, Bagheri B, Habibi V (2013) Coincidence of total anomalous pulmonary venous drainage to the superior vena cava, common atrium, and single ventricle: A very rare condition. Echocardiography 30: E98-E101.

16. Rastelli GC, Rahimtoola SH, Ongley PA, McGoon DC (1968) Common atrium: Anatomy, hemodynamics, and surgery. J Thorac Cardiovasc Surg 55: 834-841.

17. Munoz-Armas S, Gorrin JR, Anselmi G, Hernández PB, Anselmi A (1968) Single atrium: Embryologic, anatomic, electrocardiographic and other diagnostic features. Am J Cardiol 21: 639-652.

18. Abdullah NL, Quek SC, Seto KY, Teo LL (2015) Clinics in diagnostic imaging (160). Levocardia with abdominal situs inversus. Singapore Med J 56: 198-201.
19. Tomita H, Yamada O, Ohuchi H, Ono $Y$, Arakaki $Y$, et al. (2001) Coagulation profile, hepatic function, and hemodynamics following Fontan-type operations. Cardiol Young 11: 62-66.

20. Ravn HB, Hjortdal VE, Stenbog EV, Emmertsen K, Kromann O, et al. (2001) Increased platelet reactivity and significant changes in coagulation markers after cavopulmonary connection. Heart 85: 61-65.

21. Ghaferi AA, Hutchins GM (2005) Progression of liver pathology in patients undergoing the Fontan procedure: Chronic passive congestion, cardiac cirrhosis, hepatic adenoma, and hepatocellular carcinoma. J Thorac Cardiovasc Surg 129: 1348-1352.

22. Gamillscheg A, Beitzke A, Stein JI, Rupitz M, Zobel G, et al. (1998) Trans-catheter coil occlusion of residual interatrial communications after Fontan procedure. Heart 80: 49-53. 